\title{
Discrete Approximations of Strong Solutions of Reflecting SDEs with Discontinuous Coefficients
}

by

\author{
Alina SEMRAU \\ Presented by Jerzy $Z A B C Z Y K$
}

Summary. We study $L^{p}$ convergence for the Euler scheme for stochastic differential equations reflecting on the boundary of a general convex domain $D \subseteq \mathbb{R}^{d}$. We assume that the equation has the pathwise uniqueness property and its coefficients are measurable and continuous almost everywhere with respect to the Lebesgue measure. In the case $D=[0, \infty)$ new sufficient conditions ensuring pathwise uniqueness for equations with possibly discontinuous coefficients are given.

1. Introduction. In this paper we investigate $L^{p}$ convergence of timediscretization schemes to solutions of $d$-dimensional stochastic differential equations (SDEs) on a convex domain $D \subseteq \mathbb{R}^{d}$ with reflecting boundary condition of the form

$$
X_{t}=X_{0}+\int_{0}^{t} \sigma\left(X_{s}\right) d W_{s}+\int_{0}^{t} b\left(X_{s}\right) d s+K_{t}, \quad t \geq 0 .
$$

Here $X_{0}=x_{0} \in \bar{D}=D \cup \partial D, X$ is a reflecting process on $\bar{D}, K$ is a bounded variation process with variation $|K|$ increasing only when $X_{t} \in \partial D, W$ is a $d$-dimensional standard Wiener process and $\sigma: \bar{D} \rightarrow \mathbb{R}^{d} \otimes \mathbb{R}^{d}, b: \bar{D} \rightarrow \mathbb{R}^{d}$ are measurable functions.

We mainly concentrate on the Euler scheme defined by the following recurrent formula:

$$
\bar{X}_{0}^{n}=X_{0}, \quad \bar{X}_{(k+1) / n}^{n}=\Pi\left(\bar{X}_{k / n}^{n}+b\left(\bar{X}_{k / n}^{n}\right) \frac{1}{n}+\sigma\left(\bar{X}_{k / n}^{n}\right) \Delta W_{k+1}\right)
$$

2000 Mathematics Subject Classification: 60H20, 60H99, 60F17.

Key words and phrases: stochastic differential equation, reflecting boundary condition, Skorokhod problem, pathwise uniqueness. 
and $\bar{X}_{t}^{n}=\bar{X}_{k / n}^{n}$ for $t \in[k / n,(k+1) / n), k \in \mathbb{N} \cup\{0\}, n \in \mathbb{N}$, where $\Pi(x)$ is the projection of $x$ on $\bar{D}$.

Let us remark that some theorems on convergence of (1.2) appeared earlier in Chitashvili and Lazrieva (1981), Słomiński (1994) and Petterson (1995). However, these results are proved under rather restrictive conditions like boundedness of the domain, condition $(\beta)$ introduced by Tanaka (1979) as well as boundedness and Lipschitz continuity of coefficients. The paper of Słomiński (2001) yields the convergence of $\left\{\bar{X}^{n}\right\}$ in $L^{p}$ whenever $D$ is a general convex domain in $\mathbb{R}^{d}$, the coefficients $\sigma, b$ of (1.1) are continuous and the SDE (1.1) has the pathwise uniqueness property. The continuity condition is omitted in Gyöngy and Krylov (1996) but they consider the equation without reflecting boundary.

In this paper we assume that (1.1) is pathwise unique and $\sigma: \bar{D} \rightarrow$ $\mathbb{R}^{d} \otimes \mathbb{R}^{d}, b: \bar{D} \rightarrow \mathbb{R}^{d}$ are measurable functions continuous almost everywhere with respect to the Lebesgue measure, i.e.

$$
l\left(D_{\sigma \sigma^{*}}\right)=0, \quad l\left(D_{b}\right)=0,
$$

where $D_{\sigma \sigma^{*}}, D_{b}$ are the sets of discontinuity points of $\sigma \sigma^{*}$ and $b$ respectively. Moreover, $\sigma$ and $b$ satisfy two additional conditions:

$$
\begin{aligned}
\left\|\sigma \sigma^{*}(x)\right\|+|b(x)|^{2} & \leq L\left(1+|x|^{2}\right), & x & \in \mathbb{R}^{d}, \\
\left(\sigma \sigma^{*}(y) x, x\right) & \geq \lambda|x|^{2}, & x, y & \in \mathbb{R}^{d},
\end{aligned}
$$

for some constants $L, \lambda>0$. From the Yamada-Watanabe theorem (see e.g. Theorem 1.3 in Rong (2000)) it is well known that (1.4), (1.5) and the pathwise uniqueness property imply the existence of the unique strong solution of (1.1). Under these assumptions we prove here a new result on convergence in $L^{p}$ of $\left\{\bar{X}^{n}\right\}$ to $X$.

In the last section we give examples of pathwise unique reflecting SDEs with possibly discontinuous coefficients. The results are one-dimensional. The problem of pathwise uniqueness for nonreflecting SDEs was considered earlier by many authors. In particular, Nakao (1971) succeeds in solving it and omitting the assumption of the continuity of both $\sigma$ and $b$. Le Gall (1983) generalizes this fact using the local time technique. In our paper we show the pathwise uniqueness property for the reflecting SDE in the half line $D=[0, \infty)$. We assume that $\sigma$ and $b$ satisfy the linear growth condition (1.4) for $d=1, \sigma$ is uniformly positive and

$$
(\sigma(x)-\sigma(y))^{2} \leq|f(x)-f(y)|, \quad x, y \geq 0,
$$

for some bounded increasing function $f:[0, \infty) \rightarrow \mathbb{R}$. Our theorem is a generalization of the result of Zhang (1994), who requires stronger conditions on $\sigma$, namely that $\sigma$ and its first derivative are continuous. 
Let us now introduce some definitions and notations used further on. $\mathcal{D}\left(\mathbb{R}^{+}, \mathbb{R}^{d}\right)$ is the space of all mappings $x: \mathbb{R}^{+} \rightarrow \mathbb{R}^{d}$ which are right continuous and admit left-hand limits, with the Skorokhod topology $J_{1}$. Processes we consider have their trajectories in $\mathcal{D}\left(\mathbb{R}^{+}, \mathbb{R}^{d}\right)$. For a given process $X$ we write $\Delta X_{t}=X_{t}-X_{t-}, t \geq 0, \Delta X_{k+1}=X_{(k+1) / n}-X_{k / n}, k \in \mathbb{N} \cup\{0\}$, and $X^{\varrho^{n}}$ is the discretization of $X$, i.e. $X_{t}^{\varrho^{n}}=X_{k / n}$ for $t \in[k / n,(k+1) / n)$, $k \in \mathbb{N} \cup\{0\}, n \in \mathbb{N}$. If $X=\left(X^{1}, \ldots, X^{d}\right)$ is a local martingale then $[X]_{t}$ stands for $\sum_{i=1}^{d}\left[X^{i}\right]_{t}$, where $\left[X^{i}\right]$ is the quadratic variation process of $X^{i}$, $i=1, \ldots, d$. If $K=\left(K^{1}, \ldots, K^{d}\right)$ is a process with locally finite variation then $|K|_{t}=\sum_{i=1}^{d}\left|K^{i}\right|_{t}$, where $\left|K^{i}\right|_{t}$ is the total variation of $K^{i}$ on $[0, t]$. $L^{p}(Q), p \geq 1$, is the usual $L^{p}$-space with the Lebesgue measure $l$ on $Q$. $\mathbb{R}^{d} \otimes \mathbb{R}^{d}$ is the space of $d \times d$-matrices with the norm $\|\sigma\|=\left(\operatorname{tr} \sigma \sigma^{*}\right)^{1 / 2}$ and $\sigma^{*}$ is the matrix transpose to $\sigma$. We denote by $|\cdot|$ the usual Euclidean norm on $\mathbb{R}^{d}$ and $K_{R}^{d}=\left\{x \in \mathbb{R}^{d}:|x| \leq R\right\}$. For $\sigma_{n}, \sigma \in \mathbb{R}^{d} \otimes \mathbb{R}^{d}$ (or $b_{n}, b \in \mathbb{R}^{d}$ ) we write $\sigma_{n} \rightarrow \sigma$ in $L_{\text {loc }}^{d}(D)$ (or $b_{n} \rightarrow b$ in $L_{\text {loc }}^{d}(D)$ ) if and only if $\sigma_{n}^{i, j} \rightarrow \sigma^{i, j}$ in $L^{d}(K)$ (or $b_{n}^{i} \rightarrow b^{i}$ in $\left.L^{d}(K)\right), i, j=1, \ldots, d$, for every compact subset $K \subset \bar{D}$. Finally, " $\rightarrow \mathcal{D}$ " and " $\rightarrow \mathcal{P}$ " denote convergence in law and in probability respectively.

2. The Euler scheme. Here $D$ is a convex domain in $\mathbb{R}^{d}$ and $\mathcal{N}_{x}$ denotes the set of inward normal unit vectors at $x \in \partial D$ (see e.g. Semrau (2007)). Let $(\Omega, \mathcal{F}, P)$ be a probability space and $\left(\mathcal{F}_{t}\right)_{t \geq 0}$ be a filtration on $(\Omega, \mathcal{F}, P)$ satisfying the usual conditions. Let $Y$ be an $\left(\mathcal{F}_{t}\right)$-adapted semimartingale with initial value in $\bar{D}$, i.e.

$$
Y_{t}=Y_{0}+M_{t}+A_{t}, \quad t \geq 0,
$$

where $Y_{0} \in \bar{D}, M$ is an $\left(\mathcal{F}_{t}\right)$-adapted local martingale, $A$ is an $\left(\mathcal{F}_{t}\right)$-adapted process with bounded variation, $M_{0}=A_{0}=0$. Recall that a pair $(X, K)$ of $\left(\mathcal{F}_{t}\right)$-adapted processes is called a solution to the Skorokhod problem associated with $Y$ if:

(i) $X_{t}=Y_{t}+K_{t}, \quad t \geq 0$,

(ii) $X$ is $\bar{D}$-valued,

(iii) $K$ is a process with locally bounded variation such that $K_{0}=0$ and

$$
K_{t}=\int_{0}^{t} n_{s} d|K|_{s}, \quad|K|_{t}=\int_{0}^{t} \mathbf{1}_{\left\{X_{s} \in \partial D\right\}} d|K|_{s}, \quad t \geq 0,
$$

where $n_{s} \in \mathcal{N}_{X_{s}}$ if $X_{s} \in \partial D$.

Let $W$ be an $\left(\mathcal{F}_{t}\right)$-adapted Wiener process. Recall that the SDE (1.1) has a strong solution if there exists a pair $(X, K)$ of $\left(\mathcal{F}_{t}\right)$-adapted processes such that $(X, K)$ is the solution of the Skorokhod problem associated with 


$$
Y_{t}=X_{0}+\int_{0}^{t} \sigma\left(X_{s}\right) d W_{s}+\int_{0}^{t} b\left(X_{s}\right) d s, \quad t \geq 0
$$

In this section we consider the discrete Euler approximation $\left\{\bar{X}^{n}\right\}$ given by the formula (1.2). It can be defined to be a sequence of solutions of the SDE with reflecting boundary conditions of the form

$$
\bar{X}_{t}^{n}=X_{0}+\int_{0}^{t} \sigma\left(\bar{X}_{s-}^{n}\right) d W_{s}^{\varrho^{n}}+\int_{0}^{t} b\left(\bar{X}_{s-}^{n}\right) d \varrho_{s}^{n}+\bar{K}_{t}^{n}, \quad t \geq 0
$$

Here $\varrho_{t}^{n}=\max \{k / n: k \in \mathbb{N} \cup\{0\}, k / n \leq t\}$ and $W_{t}^{\varrho^{n}}$ is a discretization of $W, n \in \mathbb{N}$.

Let $\left(\mathcal{F}_{t}^{\varrho^{n}}\right)$ denote the discretization of $\left(\mathcal{F}_{t}\right)$, i.e. $\mathcal{F}_{t}^{\varrho^{n}}=\mathcal{F}_{k / n}$ for $t \in$ $[k / n,(k+1) / n), k \in \mathbb{N} \cup\{0\}, n \in \mathbb{N}$. We say that the $\operatorname{SDE}(2.1)$ has a strong solution if there exists a pair of $\left(\mathcal{F}_{t}^{\varrho^{n}}\right)$-adapted processes $\left(\bar{X}^{n}, \bar{K}^{n}\right)$ such that $\left(\bar{X}^{n}, \bar{K}^{n}\right)$ is the solution of the Skorokhod problem associated with

$$
\bar{Y}_{t}^{n}=X_{0}+\int_{0}^{t} \sigma\left(\bar{X}_{s-}^{n}\right) d W_{s}^{\varrho^{n}}+\int_{0}^{t} b\left(\bar{X}_{s-}^{n}\right) d \varrho_{s}^{n} .
$$

We can now formulate our main result.

TheOREM 2.1. Let $\left\{\left(\bar{X}^{n}, \bar{K}^{n}\right)\right\}_{n \in \mathbb{N}}$ be a sequence of solutions of the SDE (2.1) with coefficients $\sigma, b$ satisfying (1.3)-(1.5). If the SDE (1.1) is pathwise unique, then for every $p \in \mathbb{N}$,

$$
E \sup _{t \leq q}\left|\bar{X}_{t}^{n}-X_{t}\right|^{2 p} \rightarrow 0, \quad q \geq 0
$$

Proof. By Corollary 2.6 in Słomiński (2001),

$$
\sup _{n \in \mathbb{N}} E \sup _{t \leq q}\left|\bar{X}_{t}^{n}\right|^{2 p}<\infty, \quad q \geq 0, p \in \mathbb{N} .
$$

In the same way as in the proof of Theorem 2.1 in Semrau (2007), we show tightness of $\left\{\bar{X}^{n}\right\}_{n \in \mathbb{N}}$ in $\mathcal{D}\left(\mathbb{R}^{+}, \mathbb{R}^{d}\right)$. Moreover, we know that $\left|\Delta \bar{X}^{n}\right| \leq$ $\left|\Delta \bar{Y}^{n}\right|$. From Lemma A.4 of Słomiński (2001), (1.4) and (2.3), for every $q \geq 0$,

$$
\begin{aligned}
& E \sup _{t \leq q}\left|\Delta \bar{X}_{t}^{n}\right|^{2} \leq E \sup _{t \leq q}\left|\sigma\left(\bar{X}_{t-}^{n}\right)\left(W_{\varrho_{t}^{n}}-W_{\varrho_{t-}^{n}}\right)+b\left(\bar{X}_{t-}^{n}\right)\left(\varrho_{t}^{n}-\varrho_{t-}^{n}\right)\right|^{2} \\
& \leq 2\left\{d\left(E \sup _{t \leq q}\left\|\sigma \sigma^{*}\left(\bar{X}_{t-}^{n}\right)\right\|^{2}\right)^{1 / 2}\left(E\left\{\omega_{W}\left(\frac{1}{n}, q\right)\right\}^{4}\right)^{1 / 2}\right. \\
&\left.+\left(\frac{1}{n}\right)^{2} E \sup _{t \leq q}\left|b\left(\bar{X}_{t-}^{n}\right)\right|^{2}\right\}
\end{aligned}
$$




$$
\leq C_{1}\left\{\frac{\ln n}{n}\left(E \sup _{t \leq q}\left(1+\left|\bar{X}_{t-}^{n}\right|^{4}\right)\right)^{1 / 2}+\left(\frac{1}{n}\right)^{2} E \sup _{t \leq q}\left(1+\left|\bar{X}_{t-}^{n}\right|^{2}\right)\right\} \leq C_{2} \frac{\ln n}{n} .
$$

Consequently, $\lim _{n \rightarrow \infty} E \sup _{t \leq q}\left|\Delta \bar{X}_{t}^{n}\right|^{2}=0$, which implies that $\left\{\bar{X}^{n}\right\}_{n \in \mathbb{N}}$ is C-tight in $\mathcal{D}\left(\mathbb{R}^{+}, \mathbb{R}^{d}\right)$, i.e. every limit point of $\left\{\bar{X}^{n}\right\}_{n \in \mathbb{N}}$ is a process with continuous trajectories.

Our next goal is to prove that $\left\{\bar{X}^{n}\right\}_{n \in \mathbb{N}}$ converges in probability. For this purpose, according to Lemma 1.1 in Gyöngy and Krylov (1996), it suffices to show that from any two subsequences $(l) \subset(n)$ and $(m) \subset(n)$ it is possible to choose further subsequences $\left(l_{k}\right) \subset(l)$ and $\left(m_{k}\right) \subset(m)$ such that $\left(\bar{X}^{l_{k}}, \bar{X}^{m_{k}}\right) \rightarrow_{\mathcal{D}}(\widehat{X}, \widehat{X})$ in $\mathcal{D}\left(\mathbb{R}^{+}, \mathbb{R}^{2 d}\right)$, where $\widehat{X}$ is a process with continuous trajectories.

So let us take $(l) \subset(n)$ and $(m) \subset(n)$. From the above we deduce that $\left\{\left(\bar{X}^{l}, \bar{X}^{m}, W\right)\right\}_{n \in \mathbb{N}}$ is C-tight in $\mathcal{D}\left(\mathbb{R}^{+}, \mathbb{R}^{3 d}\right)$. Therefore we can choose subsequences $\left(l_{k}\right) \subset(l)$ and $\left(m_{k}\right) \subset(m)$ such that

$$
\left(\bar{X}^{l_{k}}, \bar{X}^{m_{k}}, W\right) \underset{\mathcal{D}}{\rightarrow}\left(\widehat{X}^{1}, \widehat{X}^{2}, \widehat{W}\right)
$$

in $\mathcal{D}\left(\mathbb{R}^{+}, \mathbb{R}^{3 d}\right)$, where $\widehat{X}^{1}, \widehat{X}^{2}$ are processes with continuous trajectories and $\widehat{W}$ is a Wiener processes with respect to the natural filration $\mathcal{F}^{\widehat{X}^{1}, \widehat{X}^{2}, \widehat{W}}$.

We see that $\bar{Y}^{n}$ is of the form $\bar{Y}_{t}^{n}=\bar{Y}_{0}^{n}+\bar{M}_{t}^{n}+\bar{V}_{t}^{n}$, where $\bar{M}^{n}$ is the square integrable martingale and $\bar{V}^{n}$ is the process with bounded variation. On account of (2.3), $\sup _{n \in \mathbb{N}}\left\{\left|\bar{Y}_{0}^{n}\right|+E\left(\left[\bar{M}^{n}\right]_{q}+\left|\bar{V}^{n}\right|_{q}^{2}\right)\right\}<\infty$ for all $q \geq 0$, which implies that $\left\{\bar{Y}^{n}\right\}_{n \in \mathbb{N}}$ satisfies the so called condition (UT) (see Jakubowski, Mémin and Pages (1989)) and so do its subsequences $\left\{\bar{Y}^{l_{k}}\right\}_{k \in \mathbb{N}},\left\{\bar{Y}^{m_{k}}\right\}_{k \in \mathbb{N}}$.

The task is now to prove that $\bar{Y}^{l_{k}} \rightarrow_{\mathcal{D}} \widehat{Y}^{1}$ and $\bar{Y}^{m_{k}} \rightarrow_{\mathcal{D}} \widehat{Y}^{2}$ in the Skorokhod topology $J_{1}$, where

$$
\widehat{Y}_{t}^{j}=\widehat{X}_{0}+\int_{0}^{t} \sigma\left(\widehat{X}_{s}^{j}\right) d \widehat{W}_{s}+\int_{0}^{t} b\left(\widehat{X}_{s}^{j}\right) d s, \quad j=1,2 .
$$

We only give the proof of the first convergence. The same reasoning applies to the other one.

The argument is a modification of the proof of Theorem 2.2 of Rozkosz and Słomiński (1997) and Theorem 2.1 of Semrau (2007). For every $n \in \mathbb{N}$ let $\bar{\tau}_{n}^{R}=\inf \left\{t \geq 0:\left|\bar{X}_{t}^{n}\right| \geq R\right.$ or $\left.\left|\bar{X}_{t-}^{n}\right| \geq R\right\}$ and $\widehat{\tau}^{R}=\inf \left\{t \geq 0:\left|\widehat{X}_{t}^{1}\right| \geq R\right.$ or $\left.\left|\widehat{X}_{t-}^{1}\right| \geq R\right\}, R>0$. In view of (2.4) and Proposition VI.2.12 of Jacod and Shiryaev (2003), there exists a sequence $\left\{R_{p}\right\}_{p \in \mathbb{N}}$ with $R_{p} \uparrow \infty$ such that for every $p \in \mathbb{N}$,

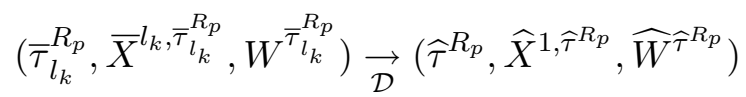

in $\mathbb{R} \times \mathcal{D}\left(\mathbb{R}^{+}, \mathbb{R}^{2 d}\right)$. 
We can construct sequences $\left\{\sigma_{i}\right\}_{i \in \mathbb{N}}$ and $\left\{b_{i}\right\}_{i \in \mathbb{N}}$ of continuous functions $\sigma_{i}: \bar{D} \rightarrow \mathbb{R}^{d} \otimes \mathbb{R}^{d}, b_{i}: \bar{D} \rightarrow \mathbb{R}^{d}$ such that $\left\|\sigma_{i} \sigma_{i}^{*}(x)\right\|+\left|b_{i}(x)\right|^{2} \leq L\left(1+|x|^{2}\right)$ for $x \in \mathbb{R}^{d}$, and $\sigma_{i} \rightarrow \sigma$ and $b_{i} \rightarrow b$ in $L_{\text {loc }}^{d}(D)$. By (2.5) and Theorem 2.6 of Jakubowski, Mémin and Pages (1989), it follows that for every $i \in \mathbb{N}$ and $p \in \mathbb{N}$,

$$
\begin{aligned}
\left(\bar{\tau}_{l_{k}}^{R_{p}}, \bar{X}^{l_{k}, \bar{\tau}_{l_{k}}^{R_{p}},}, \int_{0}^{\cdot \wedge \bar{\tau}_{l_{k}}^{R_{p}}} \sigma_{i}\left(\bar{X}_{s-}^{l_{k}}\right) d W_{s}, \quad \int_{0}^{\cdot \wedge \bar{\tau}_{l_{k}}^{R_{p}}} b_{i}\left(\bar{X}_{s-}^{l_{k}}\right) d \varrho_{s}^{l_{k}}\right) \\
\underset{\mathcal{D}}{\rightarrow}\left(\widehat{\tau}^{R_{p}}, \widehat{X}^{1, \widehat{\tau}^{R_{p}}}, \int_{0}^{\cdot \wedge \widehat{\tau}_{p}^{R_{p}}} \sigma_{i}\left(\widehat{X}_{s}^{1}\right) d \widehat{W}_{s}, \int_{0}^{\cdot \wedge \widehat{\tau}^{R_{p}}} b_{i}\left(\widehat{X}_{s}^{1}\right) d s\right)
\end{aligned}
$$

in $\mathcal{D}\left(\mathbb{R}^{+}, \mathbb{R}^{3 d}\right)$.

By the Krylov inequality for $\left\{\bar{X}^{n}\right\}_{n \in \mathbb{N}}$ and its limit from Lemma 2.2 in Semrau (2007), in a similar way to that in the proof of Theorem 2.1 in Semrau (2007) we can show that for every $\epsilon>0$ and $q \geq 0$,

$$
\begin{aligned}
& \lim _{i \rightarrow \infty} \limsup _{k \rightarrow \infty} P\left(\sup _{t \leq q}\left|\int_{0}^{t} \sigma_{i}\left(\bar{X}_{s-}^{l_{k}}\right) d W_{s}-\int_{0}^{t} \sigma\left(\bar{X}_{s-}^{l_{k}}\right) d W_{s}\right| \geq \epsilon\right)=0, \\
& \lim _{i \rightarrow \infty} \limsup _{k \rightarrow \infty} P\left(\sup _{t \leq q}\left|\int_{0}^{t} b_{i}\left(\bar{X}_{s-}^{l_{k}}\right) d \varrho^{l_{k}}-\int_{0}^{t} b\left(\bar{X}_{s-}^{l_{k}}\right) d \varrho^{l_{k}}\right| \geq \epsilon\right)=0
\end{aligned}
$$

and

$$
\begin{array}{r}
\lim _{i \rightarrow \infty} P\left(\sup _{t \leq q}\left|\int_{0}^{t} \sigma_{i}\left(\widehat{X}_{s}^{1}\right) d \widehat{W}_{s}-\int_{0}^{t} \sigma\left(\widehat{X}_{s}^{1}\right) d \widehat{W}_{s}\right| \geq \epsilon\right)=0, \\
\lim _{i \rightarrow \infty} P\left(\sup _{t \leq q}\left|\int_{0}^{t} b_{i}\left(\widehat{X}_{s}^{1}\right) d s-\int_{0}^{t} b\left(\widehat{X}_{s}^{1}\right) d s\right| \geq \epsilon\right)=0 .
\end{array}
$$

Using (2.6)-(2.10) and Theorem 3.2 of Billingsley (1999) we obtain

$$
\left(\bar{X}^{l_{k}}, \int_{0} \sigma\left(\bar{X}_{s-}^{l_{k}}\right) d W_{s}, \int_{0}^{j_{s-}} b\left(\bar{X}_{s-}^{l_{k}}\right) d \varrho_{s}^{l_{k}}\right) \underset{\mathcal{D}}{\rightarrow}\left(\widehat{X}^{1}, \int_{0}^{\infty} \sigma\left(\widehat{X}_{s}^{1}\right) d \widehat{W}_{s}, \int_{0}^{1} b\left(\widehat{X}_{s}^{1}\right) d s\right)
$$

in $\mathcal{D}\left(\mathbb{R}^{+}, \mathbb{R}^{3 d}\right)$. Using again Lemma A.4 of Słomiński (2001), (1.4) and (2.3) in much the same manner as before we find that for every $q \geq 0$,

$$
\lim _{k \rightarrow \infty} E \sup _{t \leq q}\left|\int_{0}^{t} \sigma\left(\bar{X}_{s-}^{l_{k}}\right) d W_{s}^{\varrho^{l_{k}}}-\int_{0}^{t} \sigma\left(\bar{X}_{s-}^{l_{k}}\right) d W_{s}\right|^{2}=0,
$$

and consequently

$$
\left(\bar{X}^{l_{k}}, \int_{0}^{\int_{0}} \sigma\left(\bar{X}_{s-}^{l_{k}}\right) d W_{s}^{\varrho^{l_{k}}}, \int_{0}^{\dot{1}} b\left(\bar{X}_{s-}^{l_{k}}\right) d \varrho_{s}^{l_{k}}\right) \underset{\mathcal{D}}{\longrightarrow}\left(\widehat{X}^{1}, \int_{0}^{\infty} \sigma\left(\widehat{X}_{s}^{1}\right) d \widehat{W}_{s}, \int_{0}^{\int_{0}} b\left(\widehat{X}_{s}^{1}\right) d s\right)
$$


in $\mathcal{D}\left(\mathbb{R}^{+}, \mathbb{R}^{3 d}\right)$. For this reason $\bar{Y}^{l_{k}} \rightarrow_{\mathcal{D}} \widehat{Y}^{1}$ and the same arguments give the convergence $\bar{Y}^{m_{k}} \rightarrow_{\mathcal{D}} \widehat{Y}^{2}$. Therefore, in view of Corollary A.3 in Słomiński (2001), the pairs $\left(\widehat{X}^{1}, \widehat{K}^{1}\right)$ and $\left(\widehat{X}^{2}, \widehat{K}^{2}\right)$, where $\widehat{K}^{i}=\widehat{X}^{i}-\widehat{Y}^{i}$, are two solutions to the $\operatorname{SDE}(1.1)$ with $\widehat{W}$ instead of $W$. Since the $\operatorname{SDE}(1.1)$ is pathwise unique, $\widehat{X}^{1}=\widehat{X}^{2}$, and consequently $\left\{\bar{X}^{n}\right\}_{n \in \mathbb{N}}$ converges in probability in $\mathcal{D}\left(\mathbb{R}^{+}, \mathbb{R}^{d}\right)$ to a continuous process $X$, which is the unique strong solution to the SDE (1.1). The desired conclusion now follows from (2.3).

3. The Euler-Peano scheme. In this section we consider the EulerPeano approximation for $X$. It is defined as a sequence of solutions of the SDE with reflecting boundary conditions of the form

$$
X_{t}^{n}=X_{0}+\int_{0}^{t} \sigma\left(X_{s-}^{n, \varrho^{n}}\right) d W_{s}+\int_{0}^{t} b\left(X_{s-}^{n, \varrho^{n}}\right) d s+K_{t}^{n}, \quad t \geq 0 .
$$

It is worth pointing out that we can give a simple simulating scheme associated with this approximation in the case $D=\mathbb{R}^{d-1} \times[0, \infty)$ (see Lépingle (1995)).

For this approximation one can obtain a convergence result similar to the one for $\left\{\bar{X}^{n}\right\}$ :

THeOREM 3.1. Let $\left\{\left(X^{n}, K^{n}\right)\right\}_{n \in \mathbb{N}}$ be a sequence of solutions of the SDE (3.1) with coefficients $\sigma, b$ satisfying (1.3)-(1.5). If the SDE (1.1) is pathwise unique, then for every $p \in \mathbb{N}$,

$$
E \sup _{t \leq q}\left|X_{t}^{n}-X_{t}\right|^{2 p} \rightarrow 0, \quad q \geq 0 .
$$

This can be proved quite similarly to Theorem 2.1 because Krylov's type inequality (see Lemma 2.2 in Semrau (2007)) is true with $\bar{X}^{n}$ replaced by $X^{n, \varrho^{n}}$.

4. The pathwise uniqueness property. In this section we discuss the problem of pathwise uniqueness for the equation (1.1) with possibly discontinuous coefficients in the case $D=[0, \infty)$.

The proof of the main result consists in reducing (1.1) to the equation without drift by using the Zvonkin transformation, so first consider the case $b=0$. Let $\left(X^{j}, K^{j}\right)$ satisfy

$$
X_{t \wedge \gamma^{N}}^{j}=X_{0}+\int_{0}^{t \wedge \gamma^{N}} \sigma\left(X_{s}^{j}\right) d W_{s}+K_{t \wedge \gamma^{N}}^{j}, \quad t \geq 0, j=1,2,
$$

where $\gamma^{N}=\inf \left\{t \geq 0: \max \left(X_{t}^{1}, X_{t}^{2}\right) \geq N\right\}$.

Lemma 4.1. Suppose $\sigma:[0, N] \rightarrow \mathbb{R}$ is bounded Borel measurable and satisfies the following conditions: 
(a) there exists a bounded increasing function $f:[0, N] \rightarrow \mathbb{R}$ such that for all $x, y \in[0, N]$,

$$
(\sigma(x)-\sigma(y))^{2} \leq|f(x)-f(y)|,
$$

(b) there exists a constant $\lambda>0$ such that $\sigma(x) \geq \lambda$ for all $x \in[0, N]$. Then $P\left(X_{t \wedge \gamma^{N}}^{1}=X_{t \wedge \gamma^{N}}^{2}, t \geq 0\right)=1$.

Proof. For simplicity, we write $\left(X^{j}, K^{j}\right)$ instead of $\left(X^{j, \gamma^{N}}, K^{j, \gamma^{N}}\right), j=$ 1,2 .

The proof will be divided into two steps. We first show that $L_{t}^{0}\left(X^{1}-X^{2}\right)$ $=0$ for all $t \geq 0$ a.s. The idea of the proof comes from Le Gall (1983), who obtains a similar result for the diffusion without reflecting boundary.

Let $X=X^{1}-X^{2}$ and let $\varrho:[0, \infty) \rightarrow[0, \infty)$ be defined by $\varrho(x)=x$. In view of Lemma 1.0 in Le Gall (1983), it is sufficient to show that

$$
\int_{0}^{t} \frac{\mathbf{1}_{\left(X_{s}>0\right)}}{\varrho\left(X_{s}\right)} d[X]_{s}<\infty
$$

for all $t \geq 0$, a.s.

We select a sequence $\left\{f_{n}\right\}_{n \in \mathbb{N}}$ of increasing functions such that:

(i) $f_{n} \in C^{1}(\mathbb{R}), n \in \mathbb{N}$,

(ii) $\sup _{n \in \mathbb{N}} \sup _{x \in \mathbb{R}}\left|f_{n}(x)\right| \leq M=\sup _{x \in[0, N]}|f(x)|$,

(iii) $\lim _{n \rightarrow \infty} f_{n}(x)=f(x)$ for every $x$ at which $f$ is continuous.

Analysis similar to that in the proof of Corollary 1.2 in Le Gall (1983) shows that

$$
E \int_{0}^{t} \frac{\mathbf{1}_{\left(X_{s}>0\right)}}{\varrho\left(X_{s}\right)} d[X]_{s} \leq \liminf _{n \rightarrow \infty} \int_{0}^{1} E \int_{0}^{t} f_{n}^{\prime}\left(Z_{s}^{u}\right) d s d u,
$$

where $Z^{u}=X^{2}+u\left(X^{1}-X^{2}\right)$ for $u \in[0,1]$.

By the definition of $Z^{u}$ we can briefly write

$$
Z_{t}^{u}=Z_{0}^{u}+\int_{0}^{t} \sigma^{u}\left(X_{s}^{1}, X_{s}^{2}\right) d W_{s}+K_{t}^{u}
$$

where $K^{u}=u K^{1}+(1-u) K^{2}$ and $\lambda \leq \sigma^{u} \leq K=\sup _{x \in[0, N]}|\sigma(x)|$.

The next step is to show that for every $t \geq 0$, there exists a constant $C_{3}$ such that for every $u \in[0,1]$,

$$
\sup _{a \in \mathbb{R}} E\left[L_{t}^{a}\left(Z^{u}\right)\right] \leq C_{3} .
$$

Indeed, by Tanaka's formula, 


$$
\begin{aligned}
\sup _{a \in \mathbb{R}} E\left[L_{t}^{a}\left(Z^{u}\right)\right] & \leq \sup _{a \in \mathbb{R}} E\left(|| Z_{t}^{u}-a|-| Z_{0}^{u}-a||+\left|\int_{0}^{t} \operatorname{sgn}\left(Z_{s}^{u}-a\right) d Z_{s}^{u}\right|\right) \\
& \leq E\left|Z_{t}^{u}-Z_{0}^{u}\right|+\sup _{a \in \mathbb{R}} E\left|\int_{0}^{t} \operatorname{sgn}\left(Z_{s}^{u}-a\right) d Z_{s}^{u}\right|=I_{1}+I_{2} .
\end{aligned}
$$

We estimate only $I_{2}$. The boundedness of $I_{1}$ can be obtained analogously. By virtue of Schwarz's inequality and Corollary 1 of Słomiński (1994),

$$
\begin{aligned}
I_{2} & \leq \sup _{a \in \mathbb{R}}\left(E\left|\int_{0}^{t} \operatorname{sgn}\left(Z_{s}^{u}-a\right) \sigma^{u}\left(X_{s}^{1}, X_{s}^{2}\right) d W_{s}+\int_{0}^{t} \operatorname{sgn}\left(Z_{s}^{u}-a\right) d K_{s}^{u}\right|^{2}\right)^{1 / 2} \\
& \leq\left(2 E \int_{0}^{t}\left(\sigma^{u}\left(X_{s}^{1}, X_{s}^{2}\right)\right)^{2} d s+2 E\left|u K_{t}^{1}+(1-u) K_{t}^{2}\right|^{2}\right)^{1 / 2} \\
& \leq\left(2 t K^{2}+4 u^{2} E\left|K_{t}^{1}\right|^{2}+4(1-u)^{2} E\left|K_{t}^{2}\right|^{2}\right)^{1 / 2}<\infty,
\end{aligned}
$$

which implies (4.2).

Again the same reasoning as in the proof of Corollary 1.2 in Le Gall (1983) shows that

$$
\int_{0}^{1} E \int_{0}^{t} f_{n}^{\prime}\left(Z_{s}^{u}\right) d s d u \leq \frac{2 M C_{4}}{\lambda^{2}} .
$$

Thus, $L_{t}^{0}(X)=0$ for all $t \geq 0$, a.s.

Having disposed of this preliminary step, we can now turn to showing the desired property. Set

$$
Y_{t}^{j}=X_{0}+\int_{0}^{t} \sigma\left(X_{s}^{j}\right) d W_{s}, \quad j=1,2,
$$

and $Y=Y^{1}-Y^{2}$. Applying Theorem 1 of Shashiashvili (1988) for all $t \geq 0$ we get the equality

$$
\begin{aligned}
\int_{0}^{t}\left|d\left(K_{s}^{1}-K_{s}^{2}\right)\right|= & \left|Y_{0}\right|-\left|X_{t}\right|-\int_{0}^{t} u_{s} d Y_{s}+\int_{0}^{t} \mathbf{1}_{\left(u_{s}=1\right)} d L_{s}^{0}(X) \\
& +\int_{0}^{t} \mathbf{1}_{\left(u_{s}=-1\right)} d L_{s}^{0}(-X),
\end{aligned}
$$

where $u_{t}$ is some predictable process taking the values \pm 1 . Since $L^{0}(X)=0$,

$$
E \int_{0}^{t}\left|d\left(K_{s}^{1}-K_{s}^{2}\right)\right|=-E\left|X_{t}\right| .
$$

Hence $E\left|X_{t}\right|=0$ for all $t \geq 0$, which completes the proof.

We now turn to the case $b \neq 0$ and formulate our main result. 
Theorem 4.2. Suppose $\sigma:[0, \infty) \rightarrow \mathbb{R}$ and $b:[0, \infty) \rightarrow \mathbb{R}$ are Borel measurable functions satisfying the linear growth condition (1.4) for $d=1$ and the following two assumptions:

(1) there exists a bounded increasing function $f:[0, \infty) \rightarrow \mathbb{R}$ such that for all $x, y \geq 0$,

$$
(\sigma(x)-\sigma(y))^{2} \leq|f(x)-f(y)|,
$$

(2) there exists a constant $\lambda>0$ such that $\sigma(x) \geq \lambda$ for all $x \geq 0$.

Then pathwise uniqueness holds for (1.1).

Proof. Let $\left(X^{1}, K^{1}\right),\left(X^{2}, K^{2}\right)$ be solutions of the stopped one-dimensional SDE (1.1) of the form

$$
X_{t \wedge \gamma^{N}}=X_{0}+\int_{0}^{t \wedge \gamma^{N}} \sigma\left(X_{s}\right) d W_{s}+\int_{0}^{t \wedge \gamma^{N}} b\left(X_{s}\right) d s+K_{t \wedge \gamma^{N}}, \quad t \geq 0,
$$

where $\gamma^{N}$ is defined as before. Let $h$ and $\varphi$ be functions given by

$$
h(x)=-2 \int_{0}^{x} \frac{b(y)}{\sigma^{2}(y)} d y, \quad \varphi(x)=\int_{0}^{x} e^{h(y)} d y, \quad x \in[0, N] .
$$

Since $\varphi$ is a bijection of $[0, N]$ onto $I=[0, \varphi(N)]$, we can define

$$
a(x)=\varphi^{\prime} \sigma \circ \varphi^{-1}(x), \quad x \in[0, \varphi(N)] .
$$

From Theorem 5.1 in Rozkosz and Słomiński (1997) we know that for all nonnegative measurable functions $g \in L^{1}([-N, N])$, there exists a constant $C$ depending only on $L, N$ and $t$ such that

$$
E \int_{0}^{t \wedge \gamma^{N}} g\left(X_{s}^{j}\right) d s \leq C\|g\|_{L^{1}([-N, N])}, \quad j=1,2 .
$$

Since $\varphi^{\prime}(x)$ is absolutely continuous, $\varphi^{\prime \prime}(x)$ exists almost everywhere and in addition is locally integrable. The inequality (4.4) and a reasoning similar to that in the proof of Theorem 2.10.1 in Krylov (1982) ensure that Itô's formula applies to $\varphi$ and we have

$$
\varphi\left(X_{t \wedge \gamma^{N}}^{j}\right)=\varphi\left(X_{0}\right)+\int_{0}^{t \wedge \gamma^{N}} \varphi^{\prime} \sigma\left(X_{s}^{j}\right) d W_{s}+\int_{0}^{t \wedge \gamma^{N}} \varphi^{\prime}\left(X_{s}^{j}\right) d K_{s}^{j}
$$

for $j=1,2$. Moreover, from what has been assumed about the process $K$,

$$
\begin{aligned}
\int_{0}^{t} \varphi^{\prime}\left(X_{s}^{j}\right) d K_{s}^{j} & =\int_{0}^{t} \varphi^{\prime}\left(X_{s}^{j}\right) \mathbf{1}_{\left\{X_{s}^{j}=0\right\}} d K_{s}^{j}=\int_{0}^{t} \varphi^{\prime}(0) \mathbf{1}_{\left\{X_{s}^{j}=0\right\}} d K_{s}^{j} \\
& =\int_{0}^{t} \mathbf{1}_{\left\{X_{s}^{j}=0\right\}} d K_{s}^{j}=K_{t}^{j}, \quad j=1,2 .
\end{aligned}
$$


Set $Z_{t}^{j}=\varphi\left(X_{t}^{j}\right), j=1,2$. Then (4.5) has the form

$$
Z_{t \wedge \tau^{N}}^{j}=Z_{0}+\int_{0}^{t \wedge \tau^{N}} a\left(Z_{s}^{j}\right) d W_{s}+K_{t \wedge \tau^{N}}^{j}, \quad j=1,2,
$$

where $\tau^{N}=\inf \left\{t \geq 0: \max \left(Z_{t}^{1}, Z_{t}^{2}\right) \geq \varphi(N)\right\}$. By the properties of $K$ the pair $\left(Z^{j, \tau^{N}}, K^{j, \tau^{N}}\right)$ is the solution to the Skorokhod problem associated with $U_{t \wedge \tau^{N}}^{j}=Z_{0}+\int_{0}^{t \wedge \tau^{N}} a\left(Z_{s}^{j}\right) d W_{s}, j=1,2$, and hence the solution to the SDE (4.6).

By the properties of $\sigma, \varphi^{\prime}$ and $\varphi^{-1}$ the coefficient $a$ in the equation (4.6) satisfies the assumptions (a) and (b) of Lemma 4.1, so $P\left(Z_{t \wedge \tau^{N}}^{1}=Z_{t \wedge \tau^{N}}^{2}\right.$, $t \geq 0)=1$. Since $\varphi$ is bijective,

$$
P\left(X_{t \wedge \gamma^{N}}^{1}=X_{t \wedge \gamma^{N}}^{2}, t \geq 0\right)=1
$$

and to obtain the desired conclusion it suffices to note that $\lim _{N \rightarrow \infty} \gamma^{N}=\infty$, $P$-a.s.

Corollary 4.3. Let $\sigma:[0, \infty) \rightarrow \mathbb{R}$ and $b:[0, \infty) \rightarrow \mathbb{R}$ be Borel measurable. Suppose $\sigma$ and $b$ satisfy the linear growth condition (1.4) for $d=1, \sigma$ is of bounded variation on any compact interval and there exists a constant $\lambda>0$ such that $\sigma(x) \geq \lambda$ for all $x \geq 0$. Then pathwise uniqueness holds for (1.1).

Proof. Taking $f(x)=2 K \operatorname{Var}_{[0, x]} \sigma$, where $K=\sup _{x \in[0, N]}|\sigma(x)|$, it turns out that $\sigma$ satisfies the assumptions of Lemma 4.1. Next we argue as in the proof of Theorem 4.2 .

Corollary 4.4. Let $\sigma:[0, \infty) \rightarrow \mathbb{R}$ and $b:[0, \infty) \rightarrow \mathbb{R}$ be Borel measurable. Suppose $\sigma$ and $b$ satisfy the linear growth condition (1.4) for $d=1, \sigma$ is locally Lipschitz continuous and there exists a constant $\lambda>0$ such that $\sigma(x) \geq \lambda$ for all $x \geq 0$. Then pathwise uniqueness holds for (1.1).

Proof. Since the function $a$ given by (4.3) is Lipschitz continuous and satisfies the linear growth condition, from Tanaka (1979) we have the pathwise uniqueness property for the equation (4.6), and we repeat the reasoning from the proof of Theorem 4.2.

Corollary 4.5 (Zhang (1994)). Let $\sigma:[0, \infty) \rightarrow \mathbb{R}$ and $b:[0, \infty) \rightarrow \mathbb{R}$ be Borel measurable. Suppose $\sigma$ and $b$ satisfy the linear growth condition (1.4) for $d=1, d \sigma / d x$ is continuous and there exists a constant $\lambda>0$ such that $\sigma(x) \geq \lambda$ for all $x \geq 0$. Then pathwise uniqueness holds for (1.1).

Acknowledgements. I would like to express my sincere gratitude to Professor Leszek Słomiński for his advice and suggestions. 


\section{References}

D. J. Aldous (1978), Stopping time and tightness, Ann. Probab. 6, 335-340.

P. Billingsley (1999), Convergence of Probability Measures, Wiley, New York.

R. J. Chitashvili and N. L. Lazrieva (1981), Strong solutions of stochastic differential equations with boundary conditions, Stochastics 5, 225-309.

I. Gyöngy and N. Krylov (1996), Existence of strong solutions for Itô's stochastic equations via approximations, Probab. Theory Related Fields 105, 143-158.

J. Jacod and A. N. Shiryaev (2003), Limit Theorems for Stochastic Processes, Springer, Berlin.

A. Jakubowski, J. Mémin et G. Pages (1989), Convergence en loi des suites d'intégrales stochastiques sur l'espace $D^{1}$ de Skorokhod, Probab. Theory Related Fields 81, 111137.

N. V. Krylov (1982), Controlled Diffusion Processes, Springer, New York.

J. F. Le Gall (1983), Applications du temps local aux équations différentielles stochastiques unidimensionnelles, in: Sém. de Probab. XVII, Lecture Notes in Math. 986, Springer, Berlin, 15-31.

D. Lépingle (1995), Euler scheme for reflected stochastic differential equations, Math. Comput. Simulation 38, 119-126.

S. Nakao (1972), On the pathwise uniqueness of solutions of one-dimensional stochastic differential equations, Osaka J. Math. 9, 513-518.

R. Pettersson (1995), Approximations for stochastic differential equations with reflecting convex boundaries, Stochastic Process. Appl. 59, 295-308.

S. Rong (2000), Reflecting stochastic differential equations with jumps and applications, Chapman \& Hall/CRC Res. Notes in Math. 408, Chapman \& Hall/CRC.

A. Rozkosz and L. Słomiński (1997), On stability and existence of solutions of SDEs with reflection at the boundary, Stochastic Process. Appl. 68, 285-302.

A. Semrau (2007), Euler's approximations of weak solutions of reflecting SDEs with discontinuous coefficients, Bull. Polish Acad. Sci. Math. 55, 171-182.

M. A. Shashiashvili (1988), On the variation of the difference of singular components in the Skorokhod problem and on stochastic differential systems in a half-space, Stochastics 24, 151-169.

L. Słomiński (1994), On approximation of solutions of multidimensional SDEs with reflecting boundary conditions, Stochastic Process. Appl. 50, 197-219.

L. Słomiński (2001), Euler's approximations of solutions of SDEs with reflecting boundary, ibid. 94, 317-337.

H. Tanaka (1979), Stochastic differential equations with reflecting boundary condition in convex regions, Hiroshima Math. J. 9, 163-177.

T. S. Zhang (1994), On the strong solutions of one-dimensional stochastic differential equations with reflecting boundary, Stochastic Process. Appl. 50, 135-147.

Alina Semrau

Institute of Mathematics and Physics

University of Technology and Life Sciences

Kaliskiego 7

85-796 Bydgoszcz, Poland

E-mail: alucha@utp.edu.pl

Received March 15, 2009;

received in final form July 8, 2009 